Gabriele Kettler

\title{
Die heimlichen Manager
}

Top-Sekretärinnen sagen,

was Sache ist 
Die Deutsche Bibliothek - CIP-Einheitsaufnahme

\section{Kettler, Gabriele:}

Die heimlichen Manager : Top-Sekretärinnen

sagen, was Sache ist / Gabriele Kettler. -

Wiesbaden : Gabler, 1995

ISBN 978-3-322-87139-8

ISBN 978-3-322-87138-1 (eBook)

DOI 10.1007/978-3-322-87138-1

Der Gabler Verlag ist ein Unternehmen der Bertelsmann Fachinformation.

(C) Betriebswirtschaftlicher Verlag Dr. Th. Gabler GmbH, Wiesbaden 1995

Softcover reprint of the hardcover 1st edition 1995

Lektorat: Manuela Eckstein

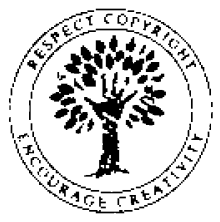

Das Werk einschließlich aller seiner Teile ist urheberrechtlich geschützt. Jede Verwertung außerhalb der engen Grenzen des Urheberrechtsgesetzes ist ohne Zustimmung des Verlags unzulässig und strafbar. Das gilt insbesondere für Vervielfältigungen, Übersetzungen, Mikroverfilmungen und die Einspeicherung und Verarbeitung in elektronischen Systemen.

Höchste inhaltliche und technische Qualität unserer Produkte ist unser Ziel. Bei der Produktion und Verbreitung unserer Bücher wollen wir die Umwelt schonen: Dieses Buch ist auf säurefreiem und chlorfrei gebleichtem Papier gedruckt. Die Einschweißfolie besteht aus Polyäthylen und damit aus organischen Grundstoffen, die weder bei der Herstellung noch bei der Verbrennung Schadstoffe freisetzen.

Die Wiedergabe von Gebrauchsnamen, Handelsnamen, Warenbezeichnungen usw. in diesem Werk berechtigt auch ohne besondere Kennzeichnung nicht zu der Annahme, daß solche Namen im Sinne der Warenzeichen- und Markenschutz-Gesetzgebung als frei zu betrachten wären und daher von jedermann benutzt werden dürften.

Redaktion: Gabriele Gerold-Schugt

Umschlaggestaltung: Schrimpf und Partner, Wiesbaden

Satz: FROMM Verlagsservice $\mathrm{GmbH}$, Selters/Ts. 


\section{Vorwort}

Sie stehen nicht im Rampenlicht, eher unter dem matten Schein einer Bürolampe, wenn alle anderen schon zu Hause vor dem Fernseher sitzen. Sie haben keine Namen, sondern bleiben anonym und nennen sich Büro von Herrn $X$ oder Sekretariat von Frau Dr. Y: Sekretärinnen, die Drahtzieher, die Ruhm und Erfolg ihrer Chefs und Chefinnen erst möglich machen - Frauen im Hintergrund, die still und leise alles vorbereiten, jeden Auftritt, jede Rede und jede Reise bis ins kleinste Detail teils in nächtelanger Kleinarbeit planen und organisieren. Sie kontrollieren das Leben der Manager, sie gehören zu ihnen wie der Dienstwagen. Doch wer sind diese Frauen, die sich um ihren Chef, dessen Wehwehchen, dessen Firma und seine ganz großen Taten mit permanentem Einsatz kümmern, aber selten Lob dafür erhalten? Sie nehmen entscheidend teil an dem Leben eines anderen, planen es und müssen immer wieder menschliche Größe zeigen, wenn es um die Lorbeerenverteilung geht. Sie bleiben ,,draußen vor der Tür“, und niemand bittet sie hinein, um den Erfolg genießen zu können. Sie arbeiten bis zum Umfallen, stehen wieder auf und sind einfach nicht totzukriegen, sind immer dort, wo man sie gerade braucht, und trotzdem wird die Außenwelt dies niemals anerkennen. Manchmal leben sie in einer Welt, die ihnen persönlich fremd ist und in der sie sich doch sicher bewegen müssen.

Ich habe mir oft Gedanken gemacht, warum das Image der Sekretärin so schlecht ist. Die Medien machen selbst in der heutigen Zeit keinen Halt vor der dummen Blondine (wahrscheinlich hat sie ein Verhältnis mit dem Chef) oder dem alles abwehrenden Zerberus im Vorzimmer. Diese Klischees sind einfach nicht auszurotten. Ich denke da nur an manche Werbesendungen: „Also, mein Chef, der ist Anwalt ..." In so manchem Film thront sie vor ihrer Schreibmaschine, telefoniert mit einer Freundin und lackiert sich dabei die Nägel. Ich finde das lächerlich und manchmal auch entwürdigend. Viele Sekretärinnen trauen sich kaum zu sagen, welchen Beruf sie ausüben. Sie geben sich als Sachbearbeiterin oder Assistentin aus.

Bevor ich meine ,Kolleginnen“ interviewte, habe ich oft gedacht, meine Erlebnisse und Eindrücke seien das Ergebnis einer verletzten Eitelkeit meinerseits. Doch ich habe feststellen müssen, daß der Gedanke, einen Beruf zweiter Klasse zu haben, bei den meisten meiner Gesprächspartnerinnen ebenfalls vorhanden war. 


\section{Vorwort}

Die Sekretärinnen haben keine Lobby, und sie sind anscheinend auch nicht wichtig genug, daß es sich lohnt, für sie das Wort zu ergreifen. Ich aber ergreife das Wort für diesen Berufsstand mit seinen auberordenttichen Frauen und möchte dazu beitragen, diesen wieder ,,gesellschaftsfähig“ zu machen. Wie hart und verantwortungsvoll dieser Beruf sein kann, weiß ich aus eigener langjähriger Erfahrung. Diese allein war jedoch nicht ausreichend, um diesen so vielfältigen, herausfordernden und teilweise aufreibenden Beruf facettenreich darzustellen.

Mit der großen Unterstützung einiger Sekretärinnen aus dem Top-Management in Deutschland konnte dieses Buch erst entstehen. Kaum jemand kann sich vorstellen, daß die Terminabsprachen für die Gespräche bereits mit enormen zeitlichen Schwierigkeiten verbunden waren. Eine Top-Sekretärin hat nicht beliebig viel Zeit. Da es sich bei meinen Interview-Partnerinnen um Top-Sekretärinnen prominenter Persönlichkeiten aus Wirtschaft und Politik handelt, werden Personen- und Firmennamen selbstverständlich nicht genannt. Finden Sie selbst heraus, wer die wirklichen (heimlichen) Manager sind.

Königstein, im April 1995

GABRIELE KETTLER 


\section{Inhaltsverzeichnis}

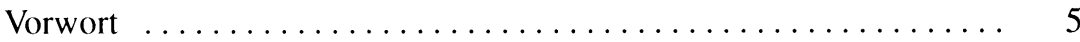

\section{Ortrud H., 44 Jahre}

Frauen braucht die Bank ......................... 9

\section{Johanna K., 43 Jahre}

Ein Karriereweg von Süd nach Nord $\ldots \ldots \ldots \ldots \ldots \ldots \ldots \ldots .23$

\section{Sybille Sch., 48 Jahre}

Eine Kostprobe aus dem Tagein-tagaus des Automobilhandels

\section{Maria M., 44 Jahre}

Politiker - eine besondere Spezies

\section{Ruth E., 57 Jahre}

Zwischen Reagenzgläsern und Aktendeckeln

\section{Barbara P., 47 Jahre}

Nie wieder Sekretärin!

\section{Sylvia St., 36 Jahre}

Freizeit - nur etwas für andere!

\section{Gabriele J., 35 Jahre}

Rund ums liebe Geld

\section{Hildegard Sch., 35 Jahre}

Auch Lehrerinnen eignen sich fürs Büro

\section{Kirsti P., 40 Jahre}

Mit Musik geht alles besser

\section{Elisabeth van R., 63 Jahre}

Vom Traum zum Traumjob 